\title{
A New Strategy for Obtaining the Pointing Stability of Stabilized Platforms
}

\author{
Mohammad Sadegh Mirzajani Darestani ${ }^{1}$, Parviz Amiri ${ }^{2}$ \\ ${ }^{1}$ Department of Electrical Engineering, Islamic Azad University of Arak, Arak, Iran \\ Mohammadsadegh.mirzajanii@gmail.com \\ ${ }^{2}$ Department of Electrical Engineering, Shahid Rajaee Teacher Training University, Tehran, Iran \\ pamiri@sru.ac.ir
}

\begin{abstract}
In this article we propose a new laboratory method for obtaining the pointing stability of a two degrees of freedom gyro stabilized platform by using a three degrees of freedom test platform. Various rated motions were applied through the three degrees of freedom test platform to the gyroscopic stabilizer and the behavior of the stabilizer to these excitations was reproduced on a checker board which placed at a distance of 7 meters from the gyroscopic stabilizer and the laser beam trajectory during the test was filmed by a video camera. The films stored in the camera for each applied motion were duly studied using MATLAB and image processing algorithms and the beam displacements from the positions marked at the outset of the test were determined. These displacements represent the pointing stability of the tested gyroscopic stabilizer in terms of degrees. Using this technique yielded a pointing stability of 0.035 degrees on the gyroscopic stabilizer in the worst case where an excitation motion with amplitude 0.093 and frequency 5.7 Hertz was applied.
\end{abstract}

Keywords: Gyroscope, Gyro stabilized Platform, Pointing Stability, Test Method.

\section{Introduction}

There are various applications in trade and industry where two degrees of freedom gyro stabilized Platform are used for imaging. In applications where these platforms are used for identifying, tracking, and taking films or photographs from the target, the camera must be sufficiently stabilized so that the required image resolution can be obtained without undue shock or vibration. Two degrees of freedom gyro stabilized platforms are generally installed inside the nose of a guiding device. While maintaining their stability, these platforms can search the space within their field of view to find their assigned targets for imaging.

The two degrees of freedom gyro stabilized platform tested in the present study included an imaging system. Therefore, the final performance criteria for the stabilizing system were defined as resolution of the image generated by the optical sensor and the allowable range for pointing parameters (regarding pointing accuracy, vibration and stability) while both the platform and the target are moving. Reduction of unstability is often considered a criterion for proper performance of the stabilizer.

The subject of two degrees of freedom gyro stabilized platforms is addressed in various sources the majority of which involve such discussions as identifying the platform system, designing the stabilizer, studying the application of such platforms in various industries, identifying the parts of a two degrees of freedom platform, etc. In [1] and [3], the structure and operation of inertial stabilized platforms were studied. In these articles, the equations of motion related to these platforms were also fully discussed. Researchers in [2] specifically studied the direct and indirect stabilization of imaging system in two axis platforms and the performance of these two methods were also discussed and compared to each other. In [4], the methods used for stabilizing electro- Optical tracking platforms were studied. This article also discussed ways of controlling the platform in the tracking mode based on fuzzy control methods. Modeling and simulation of a one axis gimbal mechanism were addressed in [5].

In an attempt to depart from the abovementioned techniques used for studying gyroscopic stabilizers, the authors endeavored to propose a laboratory testing method for evaluating the pointing stability provided by a sample two degrees of freedom gyro stabilized platform. Using this technique which is based on a three degrees of freedom test platform, we evaluated the actual operating conditions for the two degrees of freedom gyro stabilized platform as well as its 
performance under the applied conditions. The structure and performance of the stabilizer were simulated in the Matlab/Simulink and the same laboratory conditions applied to the real two degrees of freedom gyro stabilized platform by three degrees of freedom test platform were subsequently applied to the simulated structure. Thus, we successfully compared the respective behaviors of the gyroscopic stabilizer under identical working conditions in the laboratory and in the software environments.

\section{Two Degrees of Freedom Gyro Stabilized Platforms}

Gyro stabilized platforms have found widespread applications in trade and industry. Their ground, air, marine, military, and sport applications include traffic cameras, unmanned planes and aerial imaging systems installed under helicopters, automatic anti-aircraft equipment on ships, seekers installed in the leading edge of missiles, and spider cameras in sport complexes respectively. Sometimes, these platforms are used as a seeker in the leading edge of a flying machine for the purpose of tracking and following any arbitrary object. A very common application of two degrees of freedom gyro stabilized platforms is stabilizing the line of sight in a camera or an imaging element. A line of sight stabilizing system is one that includes an electro optical sensor line of sight when external disturbances are applied (such as the holder base movement) [6]. Under such conditions, when the vehicle rotates about its axes, the line of sight must remain constant relative to the inertial reference frame so that image clarity would not be lost as a result of successive vibration and shaking of the vehicle [7]. The gyroscope is used in such systems for measuring the angular velocity. Thus, two control loops are provided for searching and tracking the target. These two loops, act in opposite directions. The tracking loop purpose is to move the platform, whereas the stabilizer loop purpose is to keep the line of sight constant relative to the inertial coordinate [8]. Since the tracking and disturbance rejection have different frequency domains, these two loops can work together without interfering with each other's operation. Fig. 2 shows the control loops of a two degrees of freedom gyro stabilized platform.

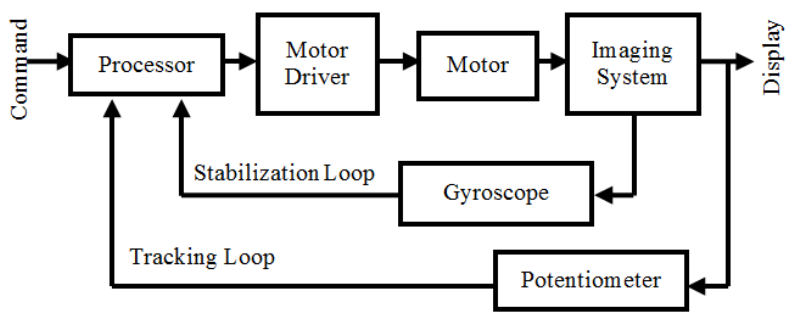

Fig. 1: Block diagram for the control loops of a two degrees of freedom gyro stabilized platform

The studied system is a two degrees of freedom gyro stabilized platform which can be arbitrarily rotated in both clock wise and counter clock wise directions and stop at a specified angle. The platform must be able to rotate the camera at constant angular velocity about either of its moving axes. For this purpose, as explained before, it is necessary to implement a precision angular velocity sensor and a proper PID controller in the velocity control loop. It is often necessary to stop the platform at a certain angular position via a PID controller and an angular position meter. The user enters the desired angle to the platform and the camera stops at entered angle upon reaching that specific angle. Two sensors are installed on each axis, one for measuring the absolute angular position relative to the mounting base and the other for measuring angular velocity relative to the inertial coordinate [9].

\section{Description and Analysis Of The Proposed Method}

As mentioned in the part II of this article, two degrees of freedom gyro stabilized platforms have two control loops, one for searching and one for tracking. Thus, it seems that the systemic tests must first identify the measurable inputs and outputs of the system and then feed certain arbitrary signals to the system to measure its corresponding outputs. On the other hand, we know from the initial data used for designing the two degrees of freedom gyro 
stabilized platform the system response form to each input or, at least, the range thereof. By comparing the actual and expected outputs, we can evaluate the general soundness of the system performance/function.

In this article, the pointing accuracy, pointing vibration, and pointing stability are introduced as the effective parameters on the pointing of a two degrees of freedom gyro stabilized platform. The proposed test and evaluation method discussed later in this article, analyze pointing stability of a sample two degrees of freedom gyro stabilized platform.

\subsection{Laser Deflection Test Equipment}

Fig. 3 shows the laser deflection test apparatus used for determining the pointing stability of the gyroscopic stabilizer. By commanding the gimbal to move to a new position as well as using the laser pointer placed on top of the gyroscopic stabilizer and its radiation on a checker board which placed at a distance of 7 meters from gyroscopic stabilizer, we can measure the pointing stability in the following manner: Various rated motions (in terms of frequency and amplitude) were applied through the three degrees of freedom test platform to the gyroscopic stabilizer and the behavior of the gyroscopic stabilizer to these excitations was reproduced on a checker board (using a laser pointer mounted on the gyroscopic stabilizer) which placed at a distance of 7 meters from the gyroscopic stabilizer and the laser beam trajectory during the test was filmed by a video camera. Upon completing the test, the films stored in the camera for each applied motion were duly studied using MATLAB and image processing algorithms and the beam displacements from the positions marked at the outset of the test were determined. These displacements represent the pointing stability of the tested gyroscopic stabilizer in terms of degrees.

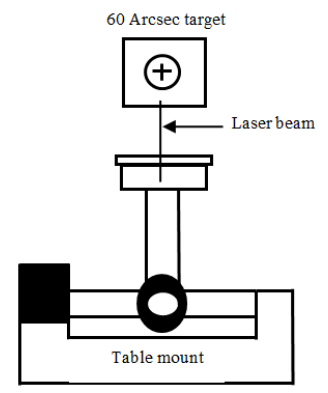

Fig. 2: Laser deflection test equipment.

\subsection{Pointing Stability}

Using laser deflection test equipment, we can measure the pointing stability of the gyroscopic stabilizer. The pointing stability is defined as the maximum deviation in the image from the actual pointing direction during a 10 seconds time interval. Fig. 4 shows this test. The desired stability for the system is 30 arc seconds. As can be seen, in Fig. 4, the pointing stability is satisfactory. 


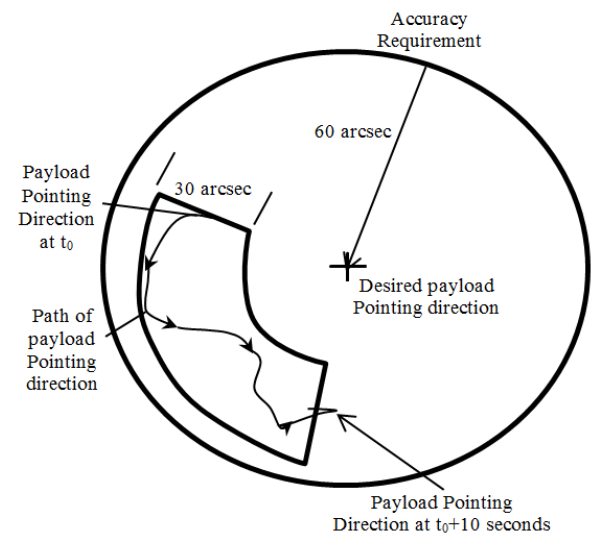

Fig. 3: The pointing stability requirement is satisfied.

\section{Measurement Results}

In this section, we obtain the pointing stability of a two degrees of freedom gyro stabilized platform using the proposed method in part IV of this article and a three degrees of freedom test platform.

The test platform has three degrees of freedom, i.e., it can rotate about the yaw, pitch, and roll axes at the desired amplitude and frequency. This advantage would enable us to generate and consequently apply the required motions and disturbances at the desired amplitudes and frequencies to the two degrees of freedom gyro stabilized platform for the purpose of evaluating its performance under various operating conditions and ensuring that it functions properly in a specific application at the required environmental and operating conditions.

In this test, Various rated motions (in terms of frequency and amplitude) were applied through the three degrees of freedom test platform to the gyroscopic stabilizer and the behavior of the gyroscopic stabilizer to these excitations was reproduced on a checker board which is positioned at a distance of 7 meters from the gyroscopic stabilizer. A video camera is used to record the laser beam trajectory on the checker board. The pointing stability of the two degrees of freedom gyro stabilized platform can be determined later on by studying the videos stored in the camera. The important point in calculating the pointing stability of the gyroscopic stabilizer is to determine the corresponding angle in degree which the two degrees of freedom gyro stabilized platform must turn to move the laser beam one division on the checker board. Equation (1) was used to obtain this Angle $(\theta)$. The distance between the checker board and the gyroscopic stabilizer (7 meters in this test) is of significance in this regard.

- Distance between two degrees of freedom gyro stabilized platform and the checker board: 7meters

- Each division width of checker board: 2.5 millimeters

$$
\theta=\arctan (25 \text { millimeters } / 7 \text { meters })=0.02 \text { degrees }
$$

Equation (1) shows that the displacement of the laser beam on the checkered board corresponds to a 0.02 degrees angular difference in the studied two degrees of freedom gyro stabilized platform. At the start of the experiment, we adjusted the two degrees of freedom gyro stabilized platform at the yaw $=0$ and $p i t c h=0$ coordinates through a computer command. Then, the desired motions with the required amplitude and frequency were applied via the three degrees of freedom test platform to the gyroscopic stabilizer. Upon applying each motion with specific amplitude and frequency, the pointing stability of gyroscopic stabilizer was obtained by counting the number of checker board divisions the laser beam was displaced on the checker board relative to the origin yaw $=0$ and pitch $=0$ and multiplying this number by 0.02 degrees. The pointing stability number was calculated in the same way for each applied motion. Finally, the greatest pointing stability number was taken as a measure and it was stated that the pointing stability number for the platform was less than the greatest pointing stability number obtained in the test. 
The pointing stability for the tested gyroscopic stabilizer was obtained for all the motions applied in Table 1 . To increase the measuring accuracy, the image processing techniques were used for analyzing the films stored by video camera for each test to obtain the pointing stability for that test. The pointing stability for each applied motion and the specifications of motions applied to the existing gyroscopic stabilizer are given in Table 1.

Table 1: The specifications of motions applied to the two degrees of freedom gyro stabilized platform and the resulting pointing stability number

\begin{tabular}{|c|c|c|c|}
\hline Item & Amplitude & Frequency (hertz) & Stability number(degree) \\
\hline 1 & 1.48 & 0.58 & 0.020 \\
\hline 2 & 0.87 & 1.16 & 0.022 \\
\hline 3 & 0.85 & 1.63 & 0.027 \\
\hline 4 & 0.6 & 2.3 & 0.028 \\
\hline 5 & 0.5 & 3 & 0.03 \\
\hline 6 & 0.35 & 3.77 & 0.01 \\
\hline 7 & 0.28 & 4.17 & 0.011 \\
\hline 8 & 0.15 & 4.9 & 0.013 \\
\hline 9 & 0.1 & 5.3 & 0.015 \\
\hline 10 & 0.093 & 5.7 & 0.018 \\
\hline
\end{tabular}

As can be seen in Table 1, the greatest stability number obtained in the test is 0.03 degrees. Therefore, the pointing stability for the tested gyroscopic stabilizer was assumed to be 0.03 degrees. The wave forms obtained in this test from the two degrees of freedom gyro stabilized platform per the 0.03 degrees stability number are as follows:

Fig. 5 shows the yaw axis motion of the gyroscopic stabilizer without drift.

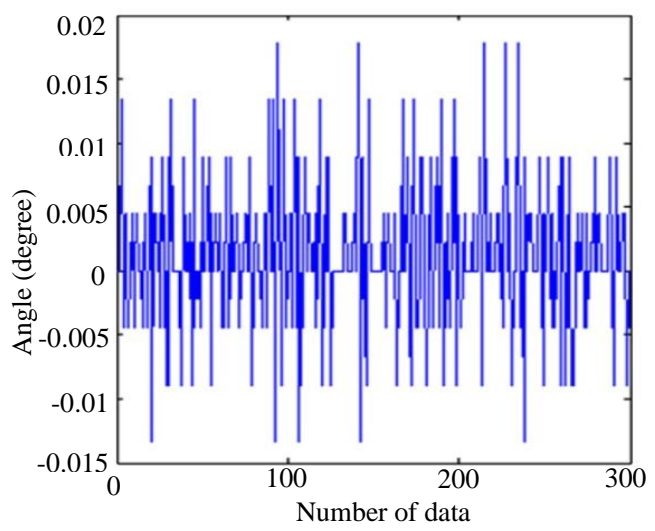

Fig. 4: The tested gyroscopic stabilizer yaw axis motion without drift.

\section{Simulation}

The structure shown in Fig. 5, was used to simulate the structure of the studied two degrees of freedom gyro stabilized platform in yaw axis. To obtain the pointing stability for the studied gyroscopic stabilizer using Matlab/Simulink, we established in the Matlab/Simulink environment conditions similar to those in the laboratory for the gyroscopic stabilizer. 


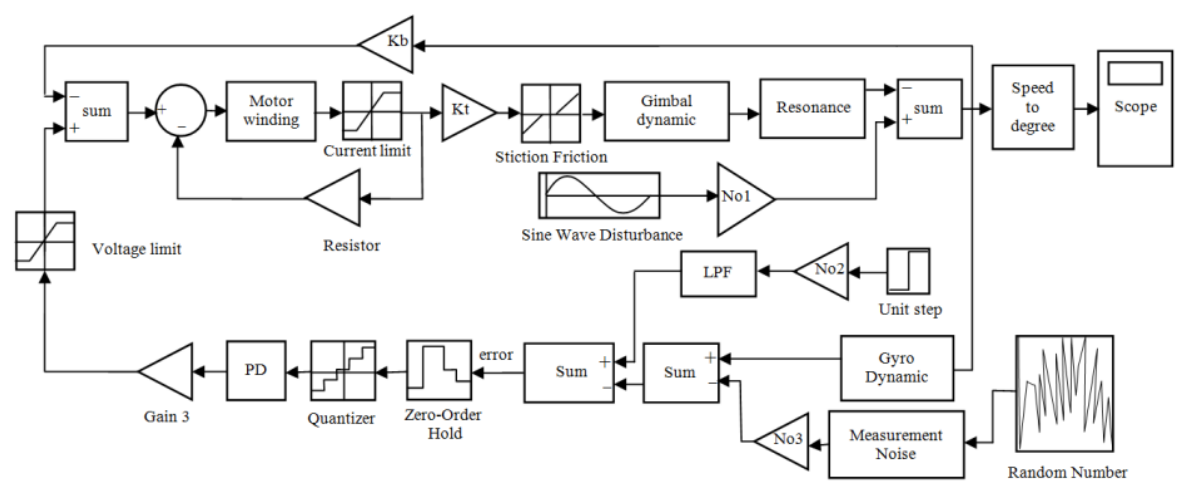

Fig. 5: The structure of the stabilized platform in yaw axis.

Fig. 6 shows the wave form of the motion undergone by the gyroscopic stabilizer in yaw axis.

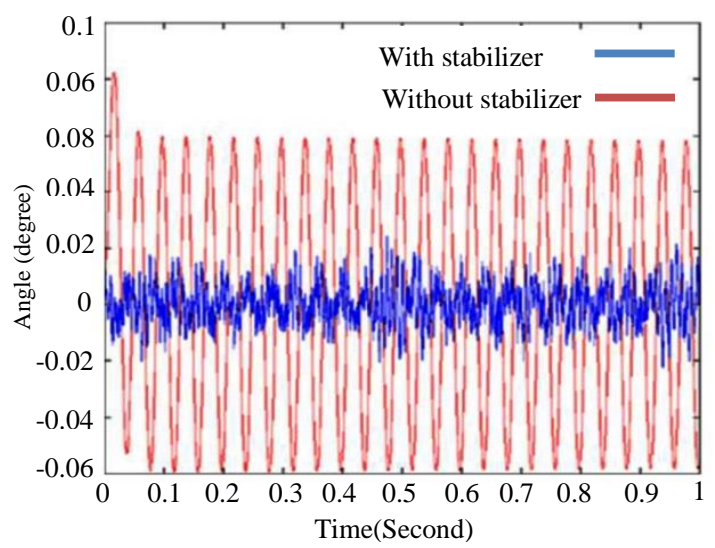

Fig. 6: Platform motion in the yaw axis with and without the stabilizer.

From the wave form shown in Fig. 6, the pointing stability of the two degrees of freedom gyro stabilized platform used for this test was obtained as 0.03 degrees which is almost equal to the pointing stability number obtained for the wave form shown in Fig. 4. Therefore, this test show that the simulation results are in good agreement with those obtained from the actual gyroscopic stabilizer test in laboratory environment.

\section{Conclusion}

The function and structure of two degrees of freedom gyro stabilized platforms, their components, and the task of each component were discussed. The proposed methods for evaluating pointing stability in this gyroscopic stabilizer were described in detail. The pointing stability of the two degrees of freedom gyro stabilized platform was measured in the worst case as 0.035 degrees upon applying a motion with an amplitude of 0.093 and frequency of 5.7 Hertz via a three degrees of freedom test platform.

\section{References}

[1] M. Masten. (2008, Feb). Inertially Stabilized Platforms for Optical Imaging Systems Tracking dynamic targets with mobile sensors. IEEE Trans. Control Systems Magazine. 28(1), pp. 47-65.

[2] P.J. Kennedy and R.L. Kennedy. (2003, Jan). Direct Versus Indirect Line of Sight (LOS) Stabilization. IEEE Trans. Control Systems Technology, 11(1), pp. 3-15. 
[3] J.M. Hilkert. (2008, Feb). Inertially Stabilized Platform Technology Concepts and Principles. IEEE Trans. Control Systems Magazine. 28(1), pp. 26-46.

[4] L. Shan-zhong and S. Long-he, "Research on Stabilizing and Tracking Control of Electro-Optical Tracking and Sighting Platform Based on Fuzzy Control," Int. Conf. on Measuring Technology and Mechatronics Automation, Changsha, china, 2010, pp. 175-178.

[5] M. Abdoa, A. Toloei, A. Vali and M. Arvan. (2014, Jan). Modeling, Control and Simulation of Cascade Control Servo System for One Axis Gimbal Mechanism. International Journal of Engineering. 27(1), pp. 157-170.

[6] W. Ji, Q. Li, B. Xu, J.j. Tu and D.a. Zhao, "Cascade Servo Control for LOS Stabilization of Opto-electronic Tracking Platform Design and Self-tuning," Int. Conf. on Information and Automation, Zhuhai, Macau, 2009, pp. 1034-1039.

[7] H. Khodadadi, M. Jahed Motlagh and M. Gorji, "Robust control and modeling a 2-DOF Inertial Stabilized Platform," Int. Conf. on Electrical, Control and Computer Engineering, Pahang, Malaysia, 2011, pp. 223-228.

[8] Z. Tao, W. Lei and W. Shu, "The Testing Techniques of Gyro Stabilized and Opto-electronic Tracking Platform," Int. Conf. on Measuring Technology and Mechatronics Automation, shanghai, china, 2009, pp. 270-273.

[9] J. Eklånge, "Design and Implementation of a Test Rig for a Gyro Stabilized Camera System," M.S. Thesis, Dept. Electrical. Eng., Linköping Univ., Linköping, Sweden, 2006. 